\title{
Lagrangian Transport and Chaotic Advection in Two-Dimensional Anisotropic Systems
}

\author{
Stephen Varghese ${ }^{1,2}$ (D) Michel Speetjens ${ }^{2} \cdot$ \\ Ruben Trieling ${ }^{1}$
}

Received: 30 August 2016/ Accepted: 25 May 2017 / Published online: 20 June 2017

(C) The Author(s) 2017. This article is an open access publication

\begin{abstract}
Enhanced geothermal systems (EGSs) are a promising concept to make geothermal power generation available in many regions worldwide. However, in current EGSs generally only a fraction of the geothermal reservoir is effectively accessed by the production fluid, resulting in suboptimal performance. Recent studies in the literature on a two-dimensional Darcy flow in a circular reservoir driven by reoriented injector-producer wells demonstrated that well configurations and pumping schemes designed on the basis of chaos theory enable efficient distribution of the production fluids throughout the entire reservoir. Key to this is accomplishment of chaotic advection, i.e. the rapid dispersion and stretching of material fluid regions, by a "proper" flow forcing. However, these studies concern isotropic reservoirs, while geothermal reservoirs typically are highly anisotropic. Our theoretical/computational study expands on said studies by investigating the impact of anisotropy on fundamental aspects of the Lagrangian transport of production fluids. This reveals that anisotropy generically eliminates key organizing mechanisms in the Lagrangian transport, viz. symmetries, and thus tends to promote disorder and, inherently, chaotic advection. However, symmetries are partially preserved — and thus order and coherence partially restored—in non-generic cases such as pumping schemes employing an even number of injector-producer wells and well configurations aligned with the anisotropy. Symmetry associated with well alignment in fact
\end{abstract}

This work is co-funded by the Foundation for Fundamental Research on Matter (FOM) of the Netherlands Organisation for Scientific Research (NWO) and Shell Global Solutions International (Grant CSER13032).

Stephen Varghese
s.varghese@tue.nl

Michel Speetjens

m.f.m.speetjens@tue.nl

Ruben Trieling

r.r.trieling@tue.nl

1 Department of Applied Physics, Technische Universiteit Eindhoven, P.O. Box 513, 5600 MB Eindhoven, The Netherlands

2 Department of Mechanical Engineering, Technische Universiteit Eindhoven, P.O. Box 513, 5600 MB Eindhoven, The Netherlands 
appears crucial to an intriguing "order within chaos" observed only in such cases: prolonged confinement of fluid to subregions of chaotic areas.

Keywords Chaotic advection · Anisotropy · Geothermal systems · Well placement · Pumping scheme

\section{Introduction}

Geothermal energy is a promising sustainable energy source and, given its insensitivity to daily and seasonal cycles, well-suited for the base load energy demand (as opposed to other sustainable solutions as e.g. wind and solar energy). Geothermal power generation is particularly interesting yet is to date mainly restricted to regions with high volcanic activity (DiPippo 2008). Enhanced geothermal systems (EGSs) offer a way to greatly expand the area of application of geothermal power generation (Brown et al. 2012). EGSs namely recover heat from hot dry rock (HDR) layers at depths typically of several kilometres through active circulation of a production fluid via injector-producer wells. The widespread presence of HDR layers makes sustainable power generation by EGSs feasible in many regions worldwide. However, despite various design and operating approaches (Grant 2011; Brown et al. 2012), EGSs to date insufficiently take fundamental transport physics into account and thus likely employ well configurations and/or pumping schemes that often are suboptimal. Field experiments by, e.g., Grant and Garg (2012), Chen and Wyborn (2009), Gérard et al. (2006) namely reveal that in current systems generally only a fraction of the geothermal reservoir is effectively accessed by the production fluid.

The above motivates our study, which aims to contribute to the optimal design and operation of EGSs by deepening insight into ways to accomplish efficient distribution of production fluids in underground reservoirs. Important to note is that, though mainly driven by EGSs, this objective is relevant in the broader scope of subsurface transport. Consider, e.g., enhanced gas and oil recovery (van Essen et al. 2009), groundwater remediation (Piscopo et al. 2013), $\mathrm{CO}_{2}$ sequestration (Pruess 2006), fracking (Zimmermann and Reinicke 2010) and heat and scalar transport in aquifers (Hidalgo et al. 2009; Bolster et al. 2011).

Recent studies by Lester et al. (2009), Metcalfe et al. (2010), Trefry et al. (2012) on the so-called rotated potential mixing (RPM) flow demonstrated that well configurations and pumping schemes designed on the basis of chaos theory enable systematic and efficient distribution of the production fluids throughout the entire reservoir. Key to this is chaotic advection, i.e. the rapid dispersion and stretching of material fluid regions, which can be accomplished by a "proper" flow forcing. This concept originates from dynamical-systems theory (Aref 1984, 2002; Ott 2002) and has found widespread application in a broad range of (practical) fluid systems (Ottino 1989; Metcalfe et al. 2012). However, the RPM flow concern isotropic reservoir conditions, while (in particular) EGSs typically consist of (manmade) fracture networks that are highly anisotropic. Our study expands on said RPM studies by investigating the impact of anisotropy on fundamental aspects of the Lagrangian transport of production fluids.

The study is organized as follows. Section 2 introduces the problem and the mathematical models. Section 3 discusses general properties and numerical treatment of the flow field. Lagrangian transport under isotropic versus anisotropic conditions is investigated in Sects. 4 and 5, respectively. Conclusions are drawn in Sect. 6. 


\section{Problem Definition}

\subsection{General Configuration}

The throughflow in a geothermal reservoir driven by reoriented injector-producer is, in accordance with Metcalfe et al. (2010), modelled by a Darcy-type dipole flow in a twodimensional (2D) circular domain of radius $R$ due to reoriented source-sink pairs. Figure 1a schematically gives the dipole flow (vectors) for the source (blue) and sink (red) located on the vertical axis. This constitutes (without loss of generality) the initial source-sink pair in this study. The full pumping scheme consists of consecutive time intervals $(k-1) T_{\text {step }} \leq t<$ $k T_{\text {step }}$, with $k=1,2, \ldots$, during which the throughflow is driven by reorientations of this source-sink pair by angle $\Theta_{k}=(k-1) \Theta$ following Fig. 1b. Step-wise reorientations $\Theta=$ $2 \pi j / N$, with $N$ and $j$ co-prime integers, are commensurate with $2 \pi$ and thus yield a timeperiodic pumping scheme with period time $T=N T_{\text {step }}$. This admits practical implementation by a static array of wells separated by an angle $\Theta$ (circles in Fig. 1b) that are pair-wise activated (red/blue in Fig. 1b). For example, Fig. 1b corresponds with reorientation $\Theta=3 \pi / 4$ and an 8 -step pumping scheme (i.e. $N=8$ ) requiring 8 wells.

Time-periodic pumping schemes result, on account of laminar flow conditions in geothermal reservoirs (Sect. 2.2), in time-periodic velocity fields $\boldsymbol{u}(\boldsymbol{x}, t+T)=u(\boldsymbol{x}, t)$ that are period-wise composed of $N$ piece-wise steady flows $\boldsymbol{u}_{k}(\boldsymbol{x})$ for each of the before-mentioned time intervals. For isotropic reservoir conditions, the reorientation in the pumping scheme carries over to the internal flow and thus renders the step-wise flows $\boldsymbol{u}_{k}$ reorientations of flow $\boldsymbol{u}_{1}$ of the first time interval following

$$
\boldsymbol{u}_{k+1}(r, \theta)=\boldsymbol{u}_{k}(r, \theta-\Theta)=\boldsymbol{u}_{1}(r, \theta-k \Theta),
$$

implying a highly-structured field with $\boldsymbol{u}_{1}$ as the steady base flow. The studies in Lester et al. (2009), Metcalfe et al. (2010), Trefry et al. (2012) concern time-periodic flows with this particular structure and reveal that the underlying reorientation is essential for the accomplishment of chaotic advection. The reorientation of the pumping scheme under anisotropic conditions no longer transfers to the internal flow and, in consequence, structure (1) is broken. This has fundamental ramifications for the Lagrangian transport properties and is the subject of investigation in the study hereafter.

\subsection{Anisotropic Reservoir Flow}

Anisotropy breaks structure (1) yet leaves the time periodicity $\boldsymbol{u}(\boldsymbol{x}, t+T)=u(\boldsymbol{x}, t)$ and the composition of step-wise steady flows $\boldsymbol{u}_{k}(\boldsymbol{x})$ intact. The present study adopts the Darcy

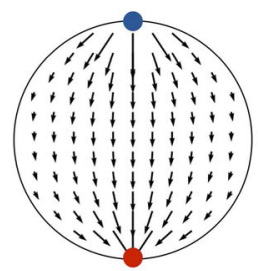

(a)

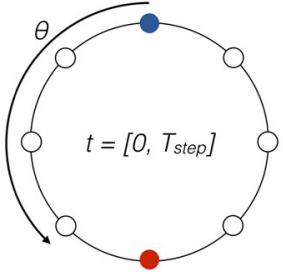

(b)

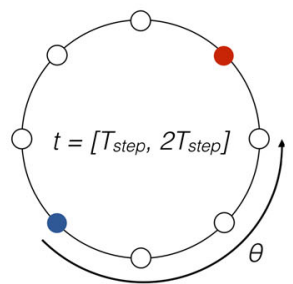

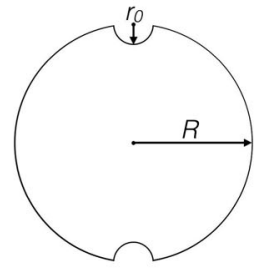

(c)

Fig. 1 General configuration: a Base flow produced by the source (blue $)$-sink (red) dipole in a 2D circular domain; b step-wise dipole reorientations by angle $\Theta$; c computational domain $\Omega_{1}$ with finite-size well pairs of radius $r_{0}$ 
Table 1 Characteristic values for physical parameters of geothermal reservoirs

\begin{tabular}{lllllll}
\hline$K\left(\mathrm{~m}^{-2}\right)$ & $\varphi(-)$ & $R(\mathrm{~m})$ & $w(\mathrm{~m})$ & $V(\mathrm{~m} / \mathrm{s})$ & $\rho_{\mathrm{f}}\left(\mathrm{kg} / \mathrm{m}^{3}\right)$ & $\mu_{@ 100^{\circ} \mathrm{C}(\mathrm{Pa} \mathrm{s})}$ \\
\hline $10^{-14}$ & $10^{-1}$ & $10^{3}$ & $10^{-3}$ & $10^{-3}$ & $10^{3}$ & $3 \times 10^{-4}$ \\
\hline
\end{tabular}

modelling approach by representing the throughflow by the so-called Darcy velocity $\boldsymbol{u}$, relating to the true velocity $\boldsymbol{v}$ within the pores via the Dupuit-Forchheimer relation $\boldsymbol{u}=\varphi \boldsymbol{v}$, with $\varphi$ the porosity (Nield and Bejan 2006). The Darcy velocity is in generic anisotropic porous media governed by the Brinkman equation

$$
\rho_{\mathrm{f}}\left[\frac{1}{\varphi} \frac{\partial \boldsymbol{u}}{\partial t}+\frac{1}{\varphi} \nabla\left(\frac{\boldsymbol{u} \cdot \boldsymbol{u}}{\varphi}\right)\right]=-\nabla p+\frac{\mu}{\varphi} \nabla^{2} \boldsymbol{u}-\mu \boldsymbol{K}^{-1} \boldsymbol{u}-c_{F} \rho_{\mathrm{f}} \boldsymbol{K}^{-1 / 2}|\boldsymbol{u}| \boldsymbol{u},
$$

with $p$ the pressure, $\rho_{\mathrm{f}}$ and $\mu$ the fluid density and viscosity, respectively, $\boldsymbol{K}$ the (to be specified) permeability tensor and $c_{\mathrm{F}}$ the Forchheimer drag coefficient (Nield and Bejan 2006). Scaling analysis using characteristic operating parameters for realistic geothermal reservoirs enables further reduction of flow model. Reservoir dimensions and corresponding permeability and porosity typically are $R \sim 1000 \mathrm{~m}, K=|\boldsymbol{K}| \sim \mathcal{O}\left(10^{-16}-10^{-12} \mathrm{~m}^{2}\right)$ and $\varphi \sim \mathcal{O}(0.1-0.4)$ (Randolph and Saar 2011; Fang et al. 2015; Xing et al. 2009; Kosack et al. 2011; Hurter and Schellschmidt 2003). Fracture widths vary over several orders of magnitudes from $w=1 \mathrm{~mm}$ to $w=100 \mathrm{~mm}$ (Li and Lior 2015; Massiot et al. 2015) and tracer-injection studies by Horne (1982), Wang et al. (2010) reveal true fluid velocities $V=|\boldsymbol{v}| \sim \mathcal{O}(0.1-10 \mathrm{~mm} / \mathrm{s})$. Based on these ranges, we choose characteristic values for the physical parameters of geothermal reservoirs as per Table 1 .

The low Reynolds and Darcy number as obtained via the characteristic values in Table 1 in conjunction with incompressibility $\boldsymbol{\nabla} \cdot \boldsymbol{u}=0$, homogenous porosity and omittance of the Forchheimer term (due to low velocities) reduces the Brinkman Eq. (2) under step-wise steady conditions to the anisotropic Darcy model

$$
\boldsymbol{u}_{k}=-\mu^{-1} \boldsymbol{K} \nabla P,
$$

for the step-wise Darcy velocities $\boldsymbol{u}_{k}$ (Nield and Bejan 2006). The permeability tensor in Eq. (3) reads

$$
\boldsymbol{K}=\boldsymbol{R} \boldsymbol{\Lambda} \boldsymbol{R}^{\mathrm{T}}=\kappa_{1} \boldsymbol{e}_{1} \boldsymbol{e}_{1}+\kappa_{2} \boldsymbol{e}_{2} \boldsymbol{e}_{2}, \quad \boldsymbol{\Lambda}=\left[\begin{array}{cc}
\kappa_{1} & 0 \\
0 & \kappa_{2}
\end{array}\right],
$$

with $\kappa_{1,2}$ the principal permeabilities along the axes delineated by the unit vectors $\boldsymbol{e}_{\mathbf{1}, \mathbf{2}}$. The latter, on account of symmetry, are orthogonal and described by

$$
\boldsymbol{e}_{1}(\alpha)=\boldsymbol{e}_{\boldsymbol{r}}(\alpha)=\boldsymbol{R}(\alpha) \boldsymbol{e}_{\boldsymbol{x}}, \quad \boldsymbol{e}_{2}(\alpha)=\boldsymbol{e}_{\boldsymbol{r}}(\alpha+\pi / 2)=\boldsymbol{R}(\alpha) \boldsymbol{e}_{\boldsymbol{y}}
$$

where $\boldsymbol{e}_{\boldsymbol{r}}(\alpha)=\cos \alpha \boldsymbol{e}_{\boldsymbol{x}}+\sin \alpha \boldsymbol{e}_{\boldsymbol{y}}$ is the radial unit vector of the polar reference frame and $\boldsymbol{R}$ is the standard rotation matrix accomplishing counter-clockwise rotation by an angle $\alpha$ about the origin.

Note that, in order to enable prescription of identical boundary conditions for any degree of anisotropy and pumping scheme, the step-wise source-sink pairs are represented by circular wells of $r_{0} \ll R$ as shown in Fig.1c which forms the domain $\Omega_{1}$ for the initial source-sink

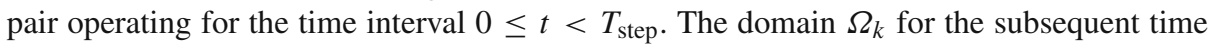
intervals $(k-1) T_{\text {step }} \leq t<k T_{\text {step }}$ is then obtained by reorienting $\Omega_{1}$ by an angle $(k-1) \Theta$. 


\subsection{Lagrangian Transport}

Lagrangian transport of the production fluid is governed by the kinematic equation

$$
\frac{\mathrm{d} \boldsymbol{x}}{\mathrm{d} t}=\boldsymbol{v}(\boldsymbol{x}, t)=\varphi^{-1} \boldsymbol{u}(\boldsymbol{x}, t),
$$

describing the position of fluid parcels $\boldsymbol{x}(t)$ at time $t$ and released at initial location $\boldsymbol{x}(0)=\boldsymbol{x}_{0}$. Here $\boldsymbol{v}$ and $\boldsymbol{u}$ are the true Eulerian velocity and associated Darcy velocity, respectively, introduced before. For time-periodic flows $\boldsymbol{v}(\boldsymbol{x}, t+T)=\boldsymbol{v}(\boldsymbol{x}, t)$, the solution to Eq. (6) is formally given by

$$
\boldsymbol{x}_{n+1}=\boldsymbol{\Phi}\left(\boldsymbol{x}_{n}\right),
$$

with $\boldsymbol{x}_{n}=\boldsymbol{x}(n T)$, constituting the stroboscopic map of the Lagrangian fluid trajectories described by (6). (Stroboscopic maps are alternatively denoted "Poincaré map" or "Poincaré section" in the literature.) Here map (7) has the composite form

$$
\boldsymbol{\Phi}=\boldsymbol{\Phi}_{N} \boldsymbol{\Phi}_{N-1} \cdots \boldsymbol{\Phi}_{1},
$$

where $\boldsymbol{\Phi}_{k}$ is the map associated with step $k$ of the pumping scheme. Reorientation property (1) translates for isotropic conditions into

$$
\boldsymbol{\Phi}_{k}=R \boldsymbol{\Phi}_{k-1} R^{-1}=R^{k-1} \boldsymbol{\Phi}_{1} R^{1-k}, \quad R: \theta \rightarrow \theta+\Theta,
$$

with $R$ the step-wise reorientation operator, tying $\boldsymbol{\Phi}_{k}$ to the base map $\boldsymbol{\Phi}_{1}$.

The combined stroboscopic map of tracers (labelling fluid parcels) released at strategic locations in the flow fully captures the Lagrangian transport in time-periodic flows (Metcalfe et al. 2012). Such maps enable identification of chaotic regions (signifying efficient transport) and transport barriers and thus facilitate systematic investigation of the transport characteristics and the effect of anisotropy.

Important is that the reservoir flows are open, i.e. tracers may enter and exit the domain through sources and sinks, respectively. Mass conservation is maintained by injection of a new tracer at the source for any tracer that leaves the domain via the sink. This injection, given that all step-wise streamlines run from source to sink, is along the same streamline via which the exit occurs. This is consistent with Metcalfe et al. (2010).

Map (7) is hereafter determined by numerical integration of kinematic Eq. (6) using a standard fourth-order Runge-Kutta method and a bilinear scheme for velocity interpolation (Kreyszig 1999). Poincaré sections are obtained by releasing 200 tracers along the symmetry lines of the flow (to be specified in Sect. 4.3).

\subsection{Non-Dimensional Form and System Parameters}

Expression of the Darcy Eq. (3) and Lagrangian equations of motion (6) and (7) in nondimensional form enables identification of the relevant system parameters. To this end the physical quantities are scaled following (primes indicate non-dimensional quantities)

$$
\boldsymbol{x}=R \boldsymbol{x}^{\prime}, \quad \boldsymbol{u}=U \boldsymbol{u}^{\prime} / 2, \quad \boldsymbol{v}=V \boldsymbol{v}^{\prime} / 2, \quad \boldsymbol{K}=\kappa_{2} \boldsymbol{K}^{\prime}, \quad p=P p^{\prime}, \quad t=\tau t^{\prime},
$$

with $\left(R, \kappa_{2}, V\right)$ as before, $U=\varphi V$ the characteristic Darcy velocity, $P=\mu U R / 2 \kappa_{2}$ the characteristic pressure and $\tau=2 R / V=2 \varphi R / U$ the characteristic time. Characteristic true velocity $V$ (and thus $U$ ) is, in accordance with Metcalfe et al. (2010), defined as the velocity at the origin of the circular reservoir. Typical values are given in Table 1 . Note that $\boldsymbol{u}=\varphi \boldsymbol{v}$ and $U=\varphi V$ implies $\boldsymbol{v}^{\prime}=\boldsymbol{u}^{\prime}$. Hence, Darcy and true velocities identify in the non-dimensional representation. 
Substituting (10) into (3) yields the non-dimensional Darcy equation

$$
\boldsymbol{u}_{k}^{\prime}=-\boldsymbol{K}^{\prime} \nabla^{\prime} p^{\prime}, \quad \boldsymbol{K}^{\prime}(\alpha, \kappa)=\boldsymbol{R} \boldsymbol{\Lambda}^{\prime} \boldsymbol{R}^{\mathrm{T}}=\kappa \boldsymbol{e}_{\mathbf{1}} \boldsymbol{e}_{\mathbf{1}}+\boldsymbol{e}_{2} \boldsymbol{e}_{2}, \quad \kappa=\frac{\kappa_{1}}{\kappa_{2}},
$$

revealing that the (step-wise) velocity field depends solely on the anisotropy via the permeability orientation $\alpha$ and the permeability ratio $\kappa$. The corresponding non-dimensional kinematic equation and stroboscopic map become

$$
\frac{\mathrm{d} \boldsymbol{x}^{\prime}}{\mathrm{d} t^{\prime}}=\boldsymbol{v}^{\prime}=\boldsymbol{u}^{\prime}, \quad \boldsymbol{x}_{n+1}^{\prime}=\boldsymbol{\Phi}^{\prime}\left(\boldsymbol{x}_{n}^{\prime}\right),
$$

with $T^{\prime}=T / \tau=V T / 2 R=U T / 2 \varphi R$ and $T_{\text {step }}^{\prime}=T_{\text {step }} / \tau=V T_{\text {step }} / 2 R=U T_{\text {step }} / 2 \varphi R$ as corresponding non-dimensional time period and step duration, respectively. The reservoir parameters in Table 1 yield $\tau \approx 20$ days. Thus $T_{\text {step }}^{\prime}=0.2$ corresponds to an operating time of $T_{\text {step }}=4$ days for one injector-producer well pair, which is practicable in actual geothermal systems.

Parameters $\left(\Theta, T_{\text {step }}^{\prime}\right)$ characterizing the pumping scheme-admitting alternative expression as $\left(\Theta, T^{\prime}\right)$ or $(\Theta, N)$-together with parameters $(\alpha, \kappa)$ characterizing the anisotropic permeability constitute the parameter space $\left(\Theta, T_{\text {step }}^{\prime}, \alpha, \kappa\right)$ for the system. The study below is performed entirely in terms of these parameters. Note that primes are dropped hereafter for brevity.

\section{Flow Field: General Properties and Numerical Treatment}

For isotropic media, in which case $\kappa=1$, the non-dimensional permeability tensor according to (11) simplifies to the identity tensor: $\boldsymbol{K}=\boldsymbol{I}$. The step-wise Darcy velocity described by (11), in consequence, becomes a potential flow: $\boldsymbol{u}_{k}=-\nabla p$. Moreover, the period-wise flow under isotropic conditions consists of reorientations of base flow $\boldsymbol{u}_{1}$ following (1). Thus the system becomes the rotated potential mixing (RPM) flow employed in the studies by Lester et al. (2009), Metcalfe et al. (2010), Trefry et al. (2012) with the base flow given analytically by

$$
\boldsymbol{u}_{1}=\left(u_{x}, u_{y}\right)=\left(\frac{4 r^{2} \cos \theta \sin \theta}{\left(1-r^{2}\right)^{2}+(2 r \cos \theta)^{2}}, \frac{-2-2 r^{2} \cos ^{2} \theta+2 r^{2} \sin ^{2} \theta}{\left(1-r^{2}\right)^{2}+(2 r \cos \theta)^{2}}\right) .
$$

The RPM flow serves as reference for the investigation of the impact of anisotropy on the Lagrangian transport properties.

For anisotropic media, two complications come into play in that (i) analytical solutions comparable to (13) are no longer available and (ii) the reorientation property (1) breaks down. Hence, determination of the (step-wise) flow $\boldsymbol{u}_{k}$ requires numerical simulation of the Darcy eq. (11) for each step of the pumping scheme. To this end we use the commercial flow solver Fluent with Dirichlet boundary conditions for $\boldsymbol{u}_{k}$ according to (13) prescribed on the step-wise finite-size source-sink pair introduced in Sect. 2.1 for $r_{0}=0.1$. This approach ensures identical step-wise boundary conditions for any pumping scheme and anisotropy. ${ }^{1}$

Fluent resolves porous-media flows via the Brinkman Eq. (2). The non-dimensional domain and corresponding Darcy Eq. (11) are implemented by defining a circular reservoir of radius $R=1 \mathrm{~m}$ with wells of radius $r_{0}=0.1 \mathrm{~m}$, prescribing boundary conditions through

\footnotetext{
1 Representation of the injector/producer wells in Fluent by a point source/sink (as in the analytical RPM flow) is not an option, since this implies locally unbounded velocities and, in consequence, results in computational instabilities.
} 


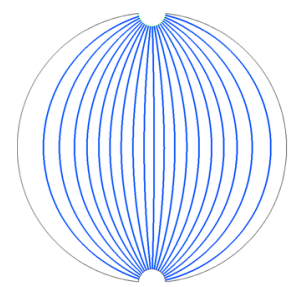

(a)

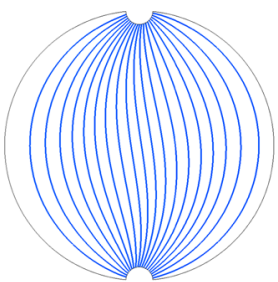

(b)

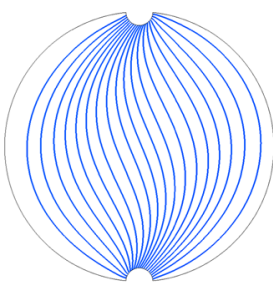

(c)

Fig. 2 Impact of anisotropy demonstrated for the streamline patterns of the first pumping step $k=1$ with permeability orientation $\alpha=\pi / 4$ and permeability ratio $\kappa$ as indicated

(13) and setting physical parameters such that the corresponding Darcy and Reynolds numbers match. ${ }^{2}$ This effectively reduces the Brinkman Eq. (2) to the Darcy Eq. (11). This is validated by comparison of the simulated flow field for isotropic conditions with the analytical RPM flow (13). This reveals a close agreement and thus signifies adequate modelling and accurate resolution of Darcy Eq. (11).

Reservoir anisotropy can vary widely, with permeability ratios ranging from the isotropic limit $\kappa=1.0$ to strong anisotropy $\kappa \sim \mathcal{O}(10)$ and basically any permeability orientation $\alpha$. Figure 2 demonstrates the impact of anisotropy on the flow field by way of the streamline patterns for the first pumping step $k=1$ with $\alpha=\pi / 4$ for increasing degree of anisotropy. This clearly reveals a progressive alignment of the streamlines with the permeability orientation $\alpha$ with growing $\kappa$ both at the injection and production wells, manifesting itself in increasingly $\mathrm{S}$-shaped streamlines that connect both wells.

\section{Lagrangian Transport in Isotropic Reservoirs}

\subsection{Lagrangian Transport Versus Pumping Scheme}

Incompressibility $\nabla \cdot \boldsymbol{u}=\boldsymbol{\nabla} \cdot \boldsymbol{v}=0$ causes the equations of motion to adopt the Hamiltonian form

$$
\frac{\mathrm{d} x}{\mathrm{~d} t}=v_{x}=\frac{\partial H}{\partial y}, \quad \frac{\mathrm{d} y}{\mathrm{~d} t}=v_{y}=-\frac{\partial H}{\partial x},
$$

with $H(\boldsymbol{x}, t)=H(\boldsymbol{x}, t+T)$ the corresponding Hamiltonian. This Hamiltonian structure has the fundamental implication that time-dependence is imperative for attaining non-trivial dynamics. Time-independence namely puts system (14) in its so-called integrable state, i.e. the state in which the Hamiltonian becomes $H=H(\boldsymbol{x})$ and acts as a stream function. Here tracers are restricted to level sets of $H$, thus ruling out chaotic advection (Aref 2002; Ott 2002). Hence, non-integrability (e.g. accomplished by time periodicity) is a necessary (yet not sufficient) condition for chaotic dynamics (Ott 2002).

Chaotic advection follows from breakdown of the integrable state through well-known Hamiltonian scenarios. For isotropic conditions, the extent of this breakdown is entirely

\footnotetext{
2 Well radius $r_{0}=0.1$ is chosen so as to preclude numerical instability and inaccuracy in Fluent due to excessive (local) velocities and at the same time minimize influence on the Lagrangian transport properties. This may suggest a significant departure from a realistic situation yet numerical investigation of the impact of well radii up to this size on the Lagrangian transport properties reveals an only marginal effect.
} 


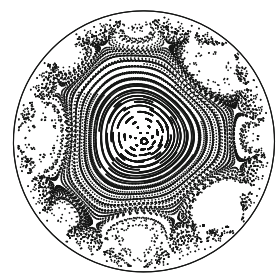

(a)

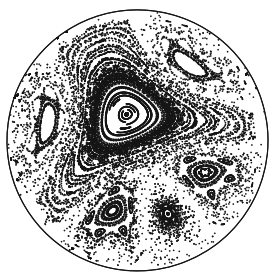

(b)

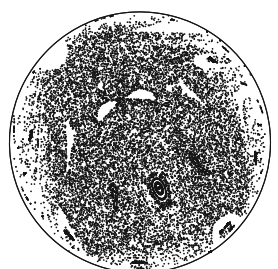

(c)

Fig. 3 Lagrangian dynamics as a function of $T_{\text {step }}$ in isotropic reservoirs demonstrated for an 8-well configuration $(\Theta=3 \pi / 4)$

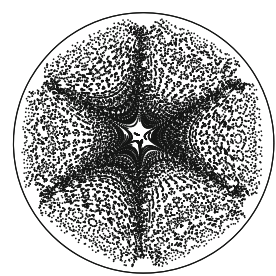

(a)

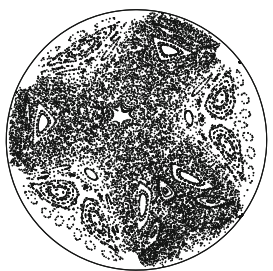

(b)

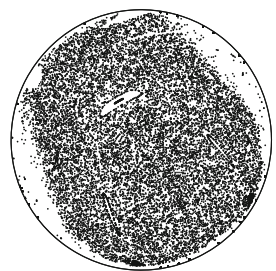

(c)

Fig. 4 Lagrangian dynamics as a function of $T_{\text {step }}$ in isotropic reservoirs demonstrated for a 3-well configuration $(\Theta=2 \pi / 3)$

dependent on the pumping scheme (parameterized by step duration $T_{\text {step }}=T / N$ and reorientation angle $\Theta$ ).

For given well configuration (i.e. given $\Theta$ ), the fluid distribution is governed solely by $T_{\text {step }}$. Figures 3 and 4, respectively, show the progression of the Lagrangian dynamics versus $T_{\text {step }}$ for well-configuration $\Theta=3 \pi / 4$ ( $N=8$ well pairs) and $\Theta=2 \pi / 3(N=3$ well pairs). This reveals a classical Hamiltonian progression in that islands (entrapment of production fluid) tend to disintegrate in favour of chaotic seas (efficient dispersion of production fluid) with increasing $T_{\text {step }}$. Hence, increasing the step duration $T_{\text {step }}$ (corresponding with a "lower" reorientation frequency between source-sink pairs) promotes the onset of chaotic advection and thus efficient fluid distribution. Conversely, decreasing $T_{\text {step }}$ ("higher" reorientation frequency) promotes the formation of islands and thus (via entrapment) impedes fluid distribution. This qualitative correlation between reorientation frequency and nature of the Lagrangian dynamics is consistent with behaviour in generic time-periodic and spatiallyperiodic flows (Aref 1984; Speetjens et al. 2006; Metcalfe et al. 2012).

The investigation below on the generic impact of anisotropy on Lagrangian transport in geothermal reservoirs concentrates on two fundamental aspects: (i) the integrable state associated with the Hamiltonian structure; (ii) symmetries in the stroboscopic map. The integrable state is the entity that must be "deconstructed" in order to accomplish chaotic advection, and thus, its structure is key to this goal. Symmetries play a central role in the generic route from integrable to chaotic state upon perturbation by time periodicity. Sections 4.2 and 4.3 elaborate on the integrable state and symmetries, respectively, under isotropic conditions. Section 5 investigates the impact of anisotropy on these elements and the generic ramifications for Lagrangian transport. 


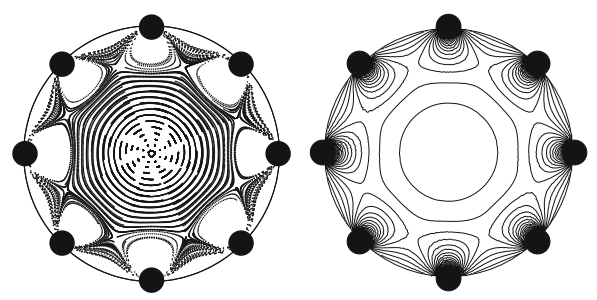

(a)

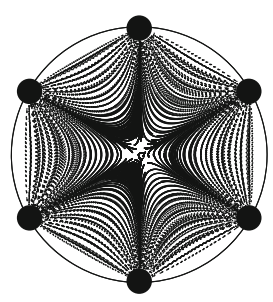

(b)

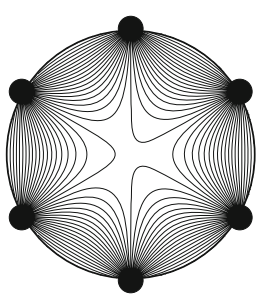

Fig. 5 Integrable limit for the Lagrangian dynamics in isotropic reservoirs: Poincaré section for $T_{\text {step }}=0.01$ (left) versus level sets of Lagrangian-averaged Hamiltonian (16) (right) for $\Theta$ as indicated and $\varepsilon=10^{-3}$

\subsection{Integrable State}

The progressions in Figs. 3 and 4 reveal the emergence of global island structures with diminishing $T_{\text {step }}$ and thus suggest that the limit of vanishing $T_{\text {step }}$ (signifying very high reorientation frequencies) results in Poincaré sections that consist entirely of islands, or equivalently, are completely devoid of chaotic regions.

It can be rigorously shown that the above behaviour is generic and limit $T_{\text {step }}=0^{+}$ (i.e. infinitesimally close yet essentially different from zero) defines the integrable state of system (14). The corresponding velocity is given by

$$
\overline{\boldsymbol{v}}\left(\boldsymbol{x}_{0}\right)=\frac{1}{N} \sum_{k=1}^{N} \boldsymbol{v}_{k}\left(\widetilde{\boldsymbol{x}}_{k}\right)=\frac{1}{N} \sum_{k=1}^{N} \boldsymbol{v}_{1}\left(R^{1-k} \widetilde{\boldsymbol{x}}_{k}\right)
$$

with

$$
\bar{H}\left(\boldsymbol{x}_{0}\right)=\frac{1}{N} \sum_{k=1}^{N} H_{k}\left(\widetilde{\boldsymbol{x}}_{k}\right)=\frac{1}{N} \sum_{k=1}^{N} H_{1}\left[R^{1-k} \widetilde{\boldsymbol{x}}_{k}\right],
$$

as associated Hamiltonian where $\widetilde{\boldsymbol{x}}_{k}$ is the step-wise initial tracer position [i.e. $\widetilde{\boldsymbol{x}}_{1}=\boldsymbol{x}_{0}$ and $\left.\tilde{\boldsymbol{x}}_{k+1}=\widetilde{\boldsymbol{x}}_{k}+\varepsilon \boldsymbol{v}_{k}\left(\widetilde{\boldsymbol{x}}_{k}\right)\right]$ ("Appendix" section).

The Hamiltonian is time-independent, i.e.

$\bar{H}=\bar{H}(\boldsymbol{x})$, and defines the before-mentioned stream function of the integrable state (Sect. 4.1). This has the fundamental implication that the sought-after integrable state indeed corresponds with limit $T_{\text {step }}=0^{+}$(and not $T_{\text {step }}=0$; "Appendix" section). The practical ramification is that a necessary (yet not sufficient) condition for chaotic advection of the production fluid is setting $T_{\text {step }}$ beyond some minimal value so as to induce disintegration of the integrable state by Hamiltonian breakdown mechanisms (Ott 2002).

Figure 5 gives $\bar{H}$ versus the Poincare section for $T_{\text {step }}=0.01$ for $\Theta$ as indicated and $\varepsilon=10^{-3}$. This demonstrates its convergence on the level sets of $\bar{H}$ with diminishing $T_{\text {step }}$. Moreover, this exposes an essential difference between pumping schemes with an even and odd number of source-sink pairs. The even case (here $\Theta=3 \pi / 4$ ) gives rise to a predominant (central) island that entraps production fluid within the reservoir and "short-circuiting zones" attached to each of the well positions (which rapidly alternate between source and sink functions). The latter consist of reconnecting streamlines via which production fluid is injected and subsequently extracted through the same well. The odd case (here $\Theta=2 \pi / 3$ ) comprises entirely of "short-circuiting zones" yet here these form between adjacent sources and sinks. 


\subsection{Symmetries}

Symmetries in the stroboscopic map are key organizing mechanisms in the Poincaré sections and thus vital to the "route to chaos" (Ottino et al. 1994; Speetjens et al. 2006; Lester et al. 2009). The symmetry analysis of the isotropic case expands on that following Lester et al. (2009). Substituting relation (9) in (8) enables expression of map $\boldsymbol{\Phi}$ entirely in terms of the base map $\boldsymbol{\Phi}_{1}$ via

$$
\boldsymbol{\Phi}=\left(R^{-1} \boldsymbol{\Phi}_{1}\right)^{N} .
$$

Symmetry of base flow $\boldsymbol{v}_{\mathbf{1}}$ yields the time-reversal reflectional symmetry

$$
\boldsymbol{\Phi}_{1}=S_{x} \boldsymbol{\Phi}_{1}^{-1} S_{x}, \quad S_{x}: \theta \rightarrow-\theta,
$$

with $S_{x}$ reflection about the $x$-axis $\mathcal{I}_{x}: \theta=0$. Furthermore, the step-wise maps relate in a time-reversal way similar to (18) via

$$
\boldsymbol{\Phi}_{2}=S_{x} \boldsymbol{\Phi}_{N}^{-1} S_{x}, \boldsymbol{\Phi}_{3}=S_{x} \boldsymbol{\Phi}_{N-1}^{-1} S_{x}, \ldots \boldsymbol{\Phi}_{N}=S_{x} \boldsymbol{\Phi}_{2}^{-1} S_{x} .
$$

The above properties result in several global symmetries of map $\boldsymbol{\Phi}$ that play a key role in the Lagrangian dynamics. This is elaborated below.

\subsubsection{Symmetry Between Sampling Levels}

Map (8) corresponds with sampling times $t_{n}=n T$, where $n=0,1, \ldots$ However, the system is periodic relative to any sequence of sampling times $t_{n}^{(i)}=n T+i T_{\text {step }}$, with $1 \leq i<N$. This admits definition of the alternative stroboscopic maps

$$
\boldsymbol{\Phi}^{(i)}=\boldsymbol{\Phi}_{N+i} \boldsymbol{\Phi}_{N-1+i} \cdots \boldsymbol{\Phi}_{1+i},
$$

where $\boldsymbol{\Phi}_{k+1}=\boldsymbol{\Phi}_{k \text { mod } N+1}$ for $k \geq N$. Maps (20) relate to "parent map" (8) via

$$
\boldsymbol{\Phi}^{(i)}=D_{i} \boldsymbol{\Phi} D_{i}^{-1}, \quad D_{i}=\boldsymbol{\Phi}_{i} \cdots \boldsymbol{\Phi}_{1},
$$

exposing $\boldsymbol{\Phi}^{(i)}$ (and thus the associated Poincaré sections) as diffeomorphisms of $\boldsymbol{\Phi}$. For isotropic conditions a second class of diffeomorphisms exist. Relation (9) namely implies $\boldsymbol{\Phi}_{k+i}=R^{i} \boldsymbol{\Phi}_{k} R^{-i}$, which enables expression of (20) as

$$
\boldsymbol{\Phi}^{(i)}=\left(R^{i} \boldsymbol{\Phi}_{N} R^{-i}\right)\left(R^{i} \boldsymbol{\Phi}_{N-1} R^{-i}\right) \cdots\left(R^{i} \boldsymbol{\Phi}_{1} R^{-i}\right)=R^{i} \boldsymbol{\Phi} R^{-i},
$$

constituting simple rotations of $(8) .^{3}$

The existence of diffeomorphisms (21) and (22) has the important practical consequence that the coherence of material regions within the production fluid (determined by the arrangement of islands and chaotic seas) is qualitatively the same at all sampling times. (Islands entrapping production fluid cannot, e.g., suddenly vanish or emerge.) Hence, the stroboscopic map $\boldsymbol{\Phi}^{(i)}$ — and thus the corresponding Poincaré sections - are reorientations of that of the original sampling times $t_{n}=n T$. This qualitative invariance in coherence-and the inherent order this imposes onto the Lagrangian dynamics - is illustrated in Fig. 6 for $\Theta=3 \pi / 4$ and $T_{\text {step }}=0.2$. Since the initial conditions for the alternative stroboscopic map $\boldsymbol{\Phi}^{(i)}$ given by $\boldsymbol{x}_{i}$ $\left(\boldsymbol{x}_{i}=\boldsymbol{\Phi}_{i} \boldsymbol{\Phi}_{i-1} \cdots \boldsymbol{\Phi}_{1} \boldsymbol{x}_{0}\right)$ are different, the Poincaré sections shown in Fig. 6 differ, though only in minute details of subsidiary islands.

\footnotetext{
3 Relations (21) and (22) do not imply that $D_{i}$ and $R^{i}$ identify; they imply the same effect.
} 


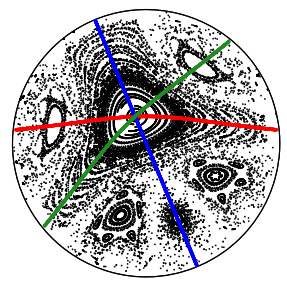

(a)

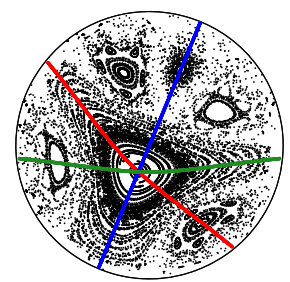

(b)

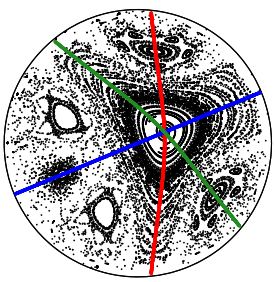

(c)

Fig. 6 Time-reversal reflectional symmetries (23) and (25) in isotropic reservoirs demonstrated for pumping scheme $\Theta=3 \pi / 4$ and $T_{\text {step }}=0.2$ by symmetry axes $\mathcal{I}_{2}$ (blue), $\mathcal{I}_{1}$ (red) and $\mathcal{I}_{1}^{\prime}$ (green) for sampling levels $t_{n}^{(i)}$ associated with (21) as indicated

\subsubsection{Symmetry Within Sampling Levels}

Substitution of (18) and (19) into (8) and employing $S_{x}^{2}=I$ and $S_{x}=S_{x}^{-1}$ yields

$$
\boldsymbol{\Phi}=S_{1} \boldsymbol{\Phi}^{-1} S_{1}^{-1}
$$

with $S_{1}=S_{x} \boldsymbol{\Phi}_{1}$. Symmetry (18) implies $S_{1}=S_{x} \boldsymbol{\Phi}_{1}=\boldsymbol{\Phi}_{1}^{-1} S_{x}=S_{1}^{-1}$ and thus $S_{1}^{2}=I$, exposing $S_{1}$ as a global time-reversal reflectional symmetry with $\mathcal{I}_{1}=S_{1} \mathcal{I}_{1}=S_{x} \boldsymbol{\Phi}_{1}^{1 / 2} \mathcal{I}_{x}$ as corresponding symmetry curve (Speetjens et al. 2006). For isotropic conditions a second time-reversal symmetry exists. Substitution of (18) in (17) through identities $R=S_{x} R^{-1} S_{x}$ and $S_{x}=R R^{-1} S_{x}$ leads to

$$
\boldsymbol{\Phi}=S_{2} \boldsymbol{\Phi}^{-1} S_{2},
$$

with $S_{2}=R^{-1} S_{x}: \theta \rightarrow-(\theta+\Theta)$. This constitutes a time-reversal reflectional symmetry with symmetry axis $\mathcal{I}_{2}=S_{2} \mathcal{I}_{2}$ given by $\mathcal{I}_{2}: \theta=-\Theta / 2$. Note that $S_{2}$ coincides with the time-reversal symmetry identified in Metcalfe et al. (2010).

Co-existence of two time-reversal reflectional symmetries, i.e. (23) and (24), implies a further time-reversal reflectional symmetry, conjugate to $S_{1}$, given by

$$
\boldsymbol{\Phi}=S_{1}^{\prime} \boldsymbol{\Phi}^{-1} S_{1}^{\prime}, \quad S_{1}^{\prime}=S_{2} S_{1} S_{2},
$$

with corresponding symmetry axis $\mathcal{I}_{1}^{\prime}=S_{2} \mathcal{I}_{1}$ the reflection of $\mathcal{I}_{1}$ about $\mathcal{I}_{2}$ (Ottino et al. 1994; Speetjens et al. 2004).

Figure 6 gives the symmetry axes $\mathcal{I}_{1}$ (red), $\mathcal{I}_{1}^{\prime}$ (green), $\mathcal{I}_{2}$ (blue) corresponding with the set of time-reversal symmetries $\left(S_{1}, S_{1}^{\prime}, S_{2}\right)$ for the Poincaré sections at $\Theta=3 \pi / 4$ and $T_{\text {step }}=0.2$. This demonstrates the strong impact of symmetries on the Lagrangian dynamics by imposing order and coherence onto the Poincaré sections. The coherent structures in the Poincaré sections namely must be either self-symmetric (if isolated) or emerge as symmetry pairs about the symmetry axes $\left(\mathcal{I}_{1}, \mathcal{I}_{1}^{\prime}, \mathcal{I}_{2}\right)$ (Speetjens et al. 2006). This property, e.g., dictates the threefold symmetry for the isolated predominant (central) island. The two upper satellite islands in Fig. 6, e.g., form a symmetric pair about $\mathcal{I}_{2}$ and are self-symmetric about either $\mathcal{I}_{1}$ or $\mathcal{I}_{1}^{\prime}$. Hence, said symmetries are key to shape and arrangement of the islands and thus play an essential role in the entrapment of production fluid. Moreover, the co-existence of multiple time-reversal symmetries implies periodic points $\boldsymbol{\Phi}(\boldsymbol{x})=\boldsymbol{x}$ at intersections of their symmetry axes (Ottino et al. 1994). Thus the elliptic point upon which the predominant island is centred, e.g. sits at intersection $\boldsymbol{x}=\mathcal{I}_{1} \cap \mathcal{I}_{2} \cap \mathcal{I}_{1}^{\prime}$. 


\section{Lagrangian Transport in Anisotropic Reservoirs}

\subsection{Hamiltonian Nature of the Lagrangian Transport}

The Hamiltonian structure of the kinematic Eq. (14) emanates solely from the incompressibility of the flow and thus is preserved under anisotropic conditions. This has the fundamental consequence that the Lagrangian transport retains the generic Hamiltonian nature as established in Sect. 4.1. Figure 7 demonstrates this by way of the progression of the Poincaré section versus the step duration $T_{\text {step }}$ for two arbitrary anisotropic cases. This reveals behaviour essentially similar to that shown in Figs. 3 and 4 for two isotropic counterparts: progressive disintegration of islands in favour of chaotic seas with increasing $T_{\text {step }}$.

The above qualitative similarity includes the convergence on an integrable state with diminishing $T_{\text {step }}$ according to Sect. 4.2. The conditions underlying the emergence of such a state namely are entirely independent of the kind of porous medium (i.e. isotropic vs. anisotropic). Isotropic and anisotropic cases differ only on a technical level in that, given the reorientation property (1) breaks down for the latter, the Hamiltonian $\bar{H}\left(\boldsymbol{x}_{0}\right)$ according to (16) associated with the anisotropic integrable state is described by the generic form based on the step-wise Hamiltonians $H_{k}\left(\tilde{\boldsymbol{x}}_{k}\right)$ instead of the form incorporating reorientations of the base flow. Figure 8 gives Poincaré sections for $\Theta=3 \pi / 4$ and $T_{\text {step }}=0.01$ for anisotropy conditions $(\alpha, \kappa)$ as indicated versus the corresponding level sets of $\bar{H}$. This demonstrates that vanishing $T_{\text {step }}$ also under anisotropic conditions leads to an integrable state. Hence, the necessary (yet not sufficient) condition established in Sect. 4.2 that $T_{\text {step }}$ must exceed a threshold value in order to admit chaotic advection is upheld.

\subsection{Dependence on Pumping Scheme and Anisotropy}

The above established that Lagrangian transport-and the route to chaotic advection-is subject to one and the same generic Hamiltonian scenario. However, despite this generality,

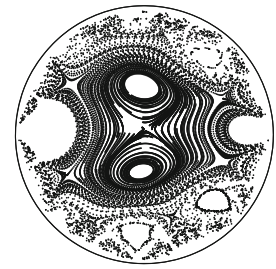

(a)

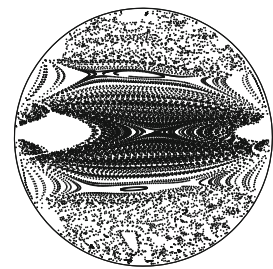

(d)

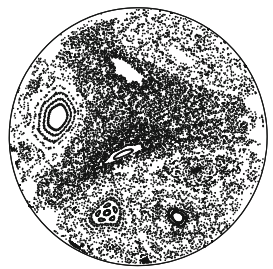

(b)

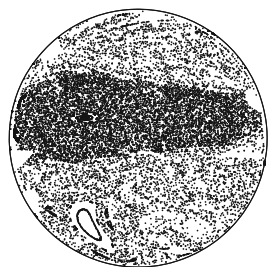

(e)



(c)

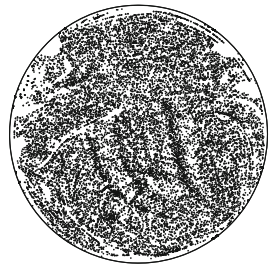

(f)

Fig. 7 Lagrangian dynamics as a function of $T_{\text {step }}$ in anisotropic reservoirs demonstrated for an 8-well configuration $(\Theta=3 \pi / 4)$ and permeability orientation $\alpha=0$ : medium anisotropy $\kappa=2$ (top) versus strong anisotropy $\kappa=10$ (bottom) 


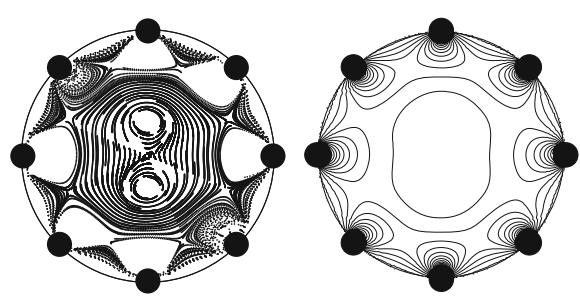

(a)

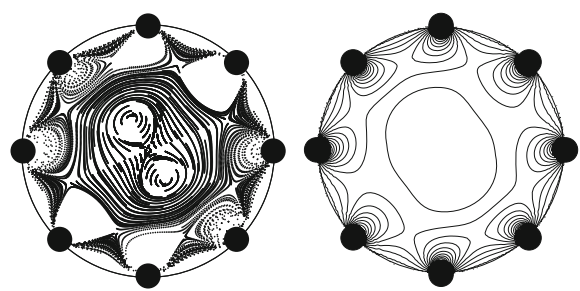

(b)

Fig. 8 Integrable limit for the Lagrangian dynamics in anisotropic reservoirs: Poincaré section for $T_{\text {step }}=$ 0.01 (left) versus level sets of Lagrangian-averaged Hamiltonian (16) (right) for $\Theta=3 \pi / 4,(\alpha, \kappa)$ as indicated and $\varepsilon=10^{-3}$

the actual Lagrangian dynamics for a given system may nonetheless depend substantially on the particular pumping scheme and anisotropy. This is investigated below through a parametric study involving the pumping scheme $\left(\Theta, T_{\text {step }}\right)$ and anisotropy parameters $(\alpha, \kappa)$.

For a given pumping scheme $\left(\Theta, T_{\text {step }}\right)$, the degree of anisotropy has a strong influence on the Lagrangian dynamics. Figure 9 demonstrates this for a number of pumping schemes specified by step duration $T_{\text {step }}=0.2$ and reorientations $\Theta=(2 \pi / 3, \pi / 6, \pi / 2,3 \pi / 4)$ (columns). The reservoir anisotropy is for each pumping scheme specified by permeability orientation $\alpha=0$ and permeability ratio $\kappa$ (rows) increasing from $\kappa=1$ (isotropic limit) to $\kappa=5$ (strongly anisotropic). This reveals that anisotropy tends to diminish the (predominant) islands of the isotropic case and thus promotes the onset of chaotic advection and, in consequence, the efficient distribution of the production fluid. However, the extent of this effect varies greatly with pumping scheme. For $\Theta=2 \pi / 3$ and $\Theta=\pi / 2$ the islands reduce dramatically or even disappear altogether; for $\Theta=\pi / 6$, on the other hand, the predominant (central) island remains largely intact. Furthermore, cases $\Theta=\pi / 2$ and (in particular) $\Theta=3 \pi / 4$ reveal that an intriguing "order within chaos" may happen within the emerging chaotic sea in that tracers are largely confined to a subregion (i.e. the "dark" region in Fig. 9k, 1 that progressively aligns with the permeability orientation (here $\alpha=0$ ). This phenomenon is examined in more detail below.

The impact of anisotropy is further investigated by way of pumping scheme $\left(\Theta, T_{\text {step }}\right)=$ $(3 \pi / 4,0.2)$. Figure 10 gives the Poincaré sections as a function of both permeability ratio $\kappa$ (rows) and permeability orientation $\alpha$ (columns). This overall confirms the above observation that anisotropy tends to promote chaotic dynamics in that the (central) islands for all cases undergo a substantial reduction with increasing $\kappa$. Moreover, these results reveal a strong influence of the permeability orientation $\alpha$ on the Lagrangian transport. Cases $\alpha=0$ and $\alpha=\pi / 4$ exhibit the abovementioned confinement of tracers to a subregion within the emerging chaotic sea that progressively aligns with the permeability orientation as $\kappa$ increases. Important to note is that these subregions are not strictly separated from the remaining parts of the chaotic sea. Tracers in fact typically visit both regions in the course of time yet residence times are substantially larger in the "dark" region. Hence, confinement is prolonged yet not indefinite. Cases $\alpha=\pi / 12$ and $\alpha=\pi / 6$, in striking contrast, are entirely devoid of such confinement, and Poincaré sections with increasing $\kappa$ develop into a chaotic sea with a uniform residence-time distribution, signifying an equal likelihood for a tracer to visit any region. An explanation for this dramatic difference in behaviour must be sought in the fact that for cases $\alpha=0$ and $\alpha=\pi / 4$, (at least) one of the source-sink well pairs of the pumping scheme always aligns with the permeability orientation. For cases $\alpha=\pi / 12$ and $\alpha=\pi / 6$, on the other hand, this does not occur. This well alignment namely has fundamental impli- 


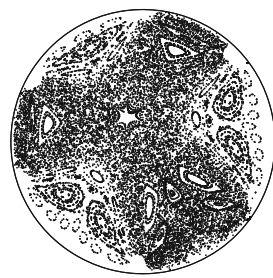

(a)

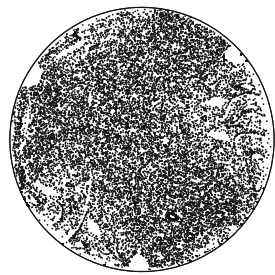

(e)

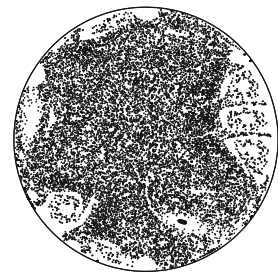

(i)

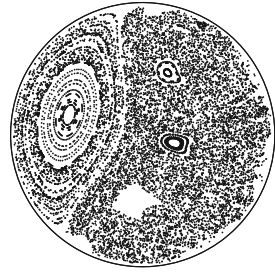

(b)

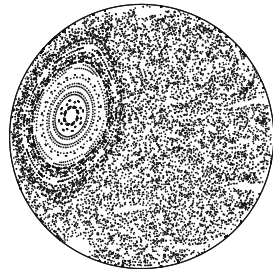

(f)

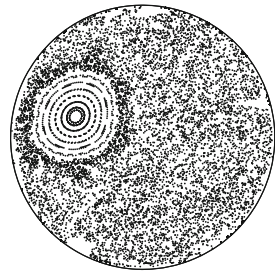

(j)

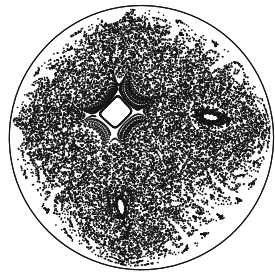

(c)

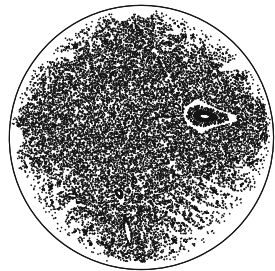

(g)

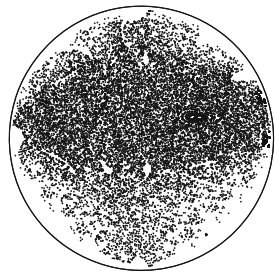

(k)

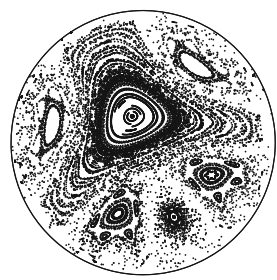

(d)

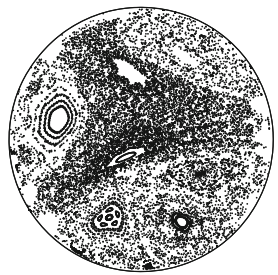

(h)

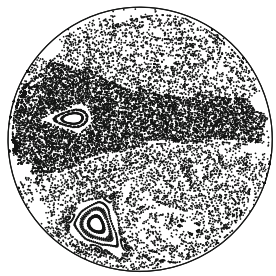

(l)

Fig. 9 Impact of anisotropy and pumping scheme on the Lagrangian dynamics demonstrated for step duration $T_{\text {step }}=0.2$ and permeability orientation $\alpha=0$ : permeability ratio $\kappa$ (rows) versus reorientation angle $\Theta$ (columns)

cations for symmetries and thus, in consequence, for the Lagrangian transport (Sects. 5.3.2 and 5.3.3).

\subsection{Symmetries}

Properties (9) and (17-19) no longer hold for generic anisotropic media and, as a consequence, the symmetries found for the isotropic case in Sect. 4.3 typically break down. Thus anisotropy eliminates important organizing mechanisms for the Lagrangian dynamics, causing a fundamental reduction in ordering and coherence within/between Poincaré sections. This is consistent with the above finding that anisotropy overall tends to diminish islands and promote chaos. However, symmetries remain important yet in a less generic way. This is elaborated below.

\subsubsection{Symmetry Between Sampling Levels}

Composition (20) of stroboscopic map $\boldsymbol{\Phi}$ and, in consequence, the corresponding diffeomorphisms $\boldsymbol{\Phi}^{(i)}$ according to (21) remain valid unconditionally for anisotropic media. Hence, the qualitative invariance of the Poincaré sections established in Sect. 4.3.1 is retained. The primary difference between isotropic and anisotropic cases is that, due to the breakdown of 


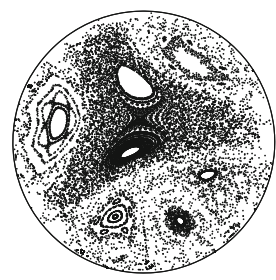

(a)

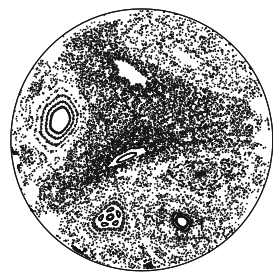

(e)

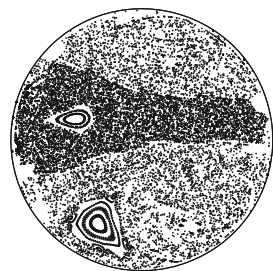

(i)



(b)

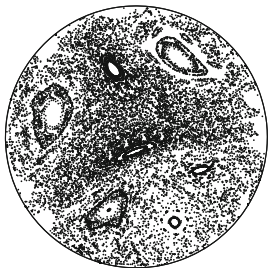

(f)

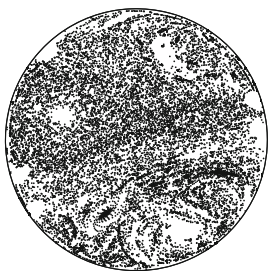

(j)

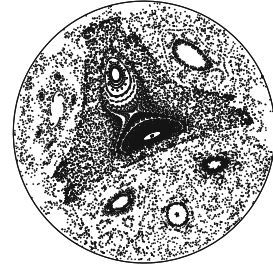

(c)

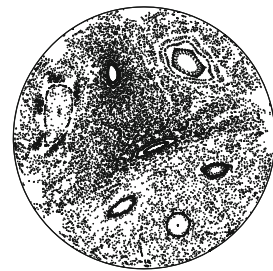

(g)

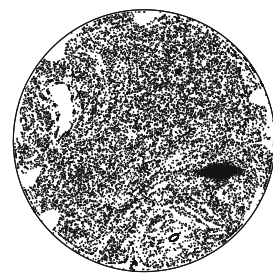

(k)

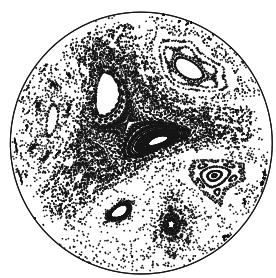

(d)

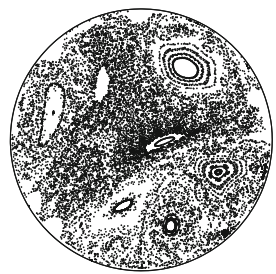

(h)

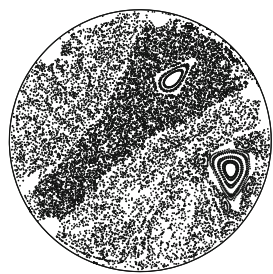

(I)

Fig. 10 Impact of anisotropy on the Lagrangian dynamics demonstrated for pumping scheme $\left(\Theta, T_{\text {step }}\right)=$ $(3 \pi / 4,0.2)$ : permeability ratio $\kappa$ (rows) versus orientation $\alpha$ (columns)

(22) for the latter, Poincaré sections at different sampling levels in general no longer relate via simple rotations.

An exception to the above exists in pumping schemes employing an even number of source-sink pairs. An even $N$ implies diagonally opposite source-sink pairs for steps $k$ and $k+N / 2$, meaning the associated step-wise maps relate via

$$
\Phi_{k+N / 2}=R_{\pi} \Phi_{k} R_{\pi}^{-1}, \quad R_{\pi}: \theta \rightarrow \theta+\pi,
$$

exposing a reorientation property akin to (9). Substituting (26) into (20) yields

$$
\boldsymbol{\Phi}^{(i+N / 2)}=R_{\pi} \boldsymbol{\Phi}^{(i)} R_{\pi}, \quad \boldsymbol{\Phi}^{(i)}=R_{\pi} \boldsymbol{\Phi}^{(i+N / 2)} R_{\pi},
$$

using $R_{\pi}^{-1}=R_{\pi}$, which constitutes a diffeomorphism similar to (22) in that Poincaré sections for $\boldsymbol{\Phi}^{(i+N / 2)}$ and $\boldsymbol{\Phi}^{(i)}$ relate via simple reorientation by $R_{\pi}$. Thus an even $N$ accomplishes a greater degree of ordering and coherence compared to odd $N$ in that effectively only $N / 2$ (instead of $N$ ) Poincaré sections relate non-trivially via diffeomorphism (21); the remaining $N / 2$ Poincaré sections are simple rotations of this set following (27). Figure 11 demonstrates property (27) for the relations $\boldsymbol{\Phi}^{(8)} \Leftrightarrow \boldsymbol{\Phi}^{(4)}$ and $\boldsymbol{\Phi}^{(5)} \Leftrightarrow \boldsymbol{\Phi}^{(1)}$ for pumping scheme $\left(\Theta, T_{\text {step }}\right)=(3 \pi / 4,0.2)$, employing $N=8$ well pairs, and $(\alpha, \kappa)=(\pi / 6,2.0)$. 


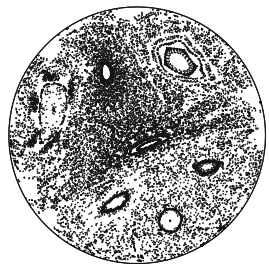

(a)

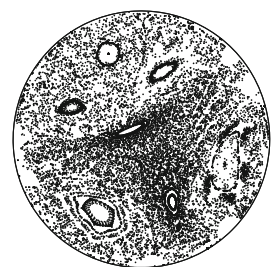

(b)

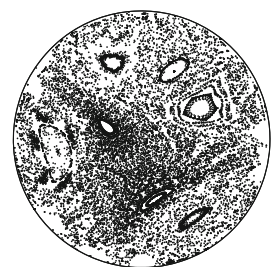

(c)

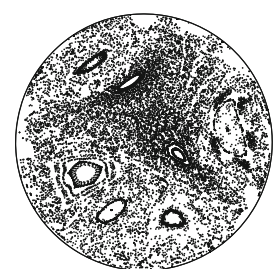

(d)

Fig. 11 Symmetry between sampling levels for even $N$ according to (27) demonstrated for pumping scheme $\left(\Theta, T_{\text {step }}\right)=(3 \pi / 4,0.2)$ and anisotropy $(\alpha, \kappa)=(\pi / 6, \kappa=2.0)$

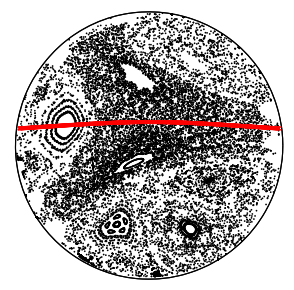

(a)

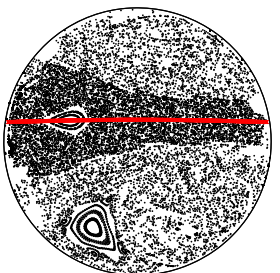

(b)

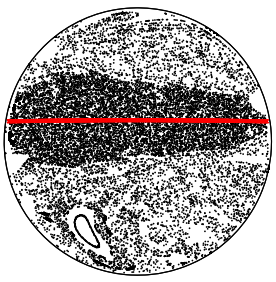

(c)

Fig. 12 Preservation of time-reversal reflectional symmetry $S_{1}$ according to (23) in anisotropic reservoirs with permeability orientations $\alpha=0$ and $\alpha=\pi / 2$ demonstrated by symmetry curve $\mathcal{I}_{1}$ (red) for $\alpha=0$, pumping scheme $\left(\Theta, T_{\text {step }}\right)=(3 \pi / 4,0.2)$ and $\kappa$ as indicated

\subsubsection{Symmetry Within Sampling Levels}

Properties (18) and (19) remain valid for anisotropic media in the special case of alignment of the first source-sink well pair with the permeability orientation (i.e. $\alpha=0$ or $\alpha=\pi / 2$ ). Hence, time-reversal reflectional symmetry $S_{1}$ according to (23) is preserved under these conditions. However, reorientation property (9) breaks down, thus eliminating the timereversal reflectional symmetry $S_{2}$ following (24) and, inherently, conjugate symmetry $S_{1}^{\prime}$ following (25). This advances $S_{1}$ as sole time-reversal symmetry for said anisotropic cases. Figure 12 illustrates symmetry $S_{1}$ by way of its symmetry curve $\mathcal{I}_{1}=S_{x} \boldsymbol{\Phi}_{1}^{1 / 2} \mathcal{I}_{x}$ (red) for pumping scheme $\left(\Theta, T_{\text {step }}\right)=(3 \pi / 4,0.2)$ and permeability direction $\alpha=0$ as a function of permeability ratio $\kappa$. This reveals a progressive concentration of the before-mentioned tracerconfinement region (Sect. 5.2) around $\mathcal{I}_{1}$, signifying an intimate relation of this phenomenon with symmetry $S_{1}$. The breakdown of reflectional symmetry $S_{2}$ in particular results in a reduced ordering and coherence in that islands off the symmetry axes must, in contrast with the isotropic case, no longer emerge in pairs. Symmetry $S_{1}$ namely admits isolated islands off $\mathcal{I}_{1}$, as evidenced in Fig. 12 by, e.g., the lower isolated island for $\kappa=5$ and $\kappa=10$. This island is invariant to symmetry $S_{1}$ in that the contained material region as whole returns to its initial position by period-wise extraction and subsequent reinjection through the wells.

Preservation of time-reversal symmetry $S_{1}$ for permeability orientation $\alpha=0$ and $\alpha=$ $\pi / 2$ generalizes to cases $\alpha_{i}=i \Theta$ and $\alpha_{i}=\pi / 2+i \Theta$, respectively, with $1 \leq i<N$, which corresponds with systems that exhibit alignment of (at least) one of the well pairs with the permeability orientation. This is elaborated in Sect. 5.3.3. 


\subsubsection{Symmetry Between Permeability Orientations}

Anisotropy introduces a new kind of symmetry that has no counterpart in isotropic media. Consider to this end map $\boldsymbol{\Phi}$ for a given well configuration $\Theta$ and given (arbitrary) permeability orientation $\alpha$. For the same well configuration $\Theta$, define $\boldsymbol{\Phi}^{[i]}$ as map associated with the set of permeability orientations $\alpha_{i}=\alpha+i \Theta$, where $1 \leq i<N$. Expressing map $\boldsymbol{\Phi}^{[i]}$ in its composite form (8) gives

$$
\boldsymbol{\Phi}^{[i]}=\boldsymbol{\Phi}_{N}^{[i]} \quad \boldsymbol{\Phi}_{N-1}^{[i]} \cdots \quad \boldsymbol{\Phi}_{1}^{[i]}
$$

where $\boldsymbol{\Phi}_{k}^{[i]}$ corresponds with step $k$ for the permeability orientation $\alpha_{i}$. The latter relates to map $\boldsymbol{\Phi}_{k}$ associated with step $k$ for the permeability orientation $\alpha$ via

$$
\boldsymbol{\Phi}_{k}^{[i]}=R^{i} \boldsymbol{\Phi}_{k-i} R^{-i}, \quad R: \theta \rightarrow \theta+\Theta,
$$

with $R$ as before. Substituting (29) in (28) ties map $\boldsymbol{\Phi}_{k}^{[i]}$ to $\boldsymbol{\Phi}^{(i)}$ following (20) via

$$
\boldsymbol{\Phi}^{[i]}=R^{i} \boldsymbol{\Phi}^{(N-i)} R^{-i}
$$

which, upon substituting (21) in (30), bridges the gap to map $\boldsymbol{\Phi}$ through

$$
\boldsymbol{\Phi}^{[i]}=R^{i} D_{N-i} \boldsymbol{\Phi} D_{N-i}^{-1} R^{-i}=G_{i} \boldsymbol{\Phi} G_{i}^{-1}, \quad G_{i}=R^{i} D_{N-i},
$$

where $1 \leq i<N$. Relation (31) reveals that, for given permeability ratio $\kappa$, the maps $\boldsymbol{\Phi}^{[i]}$ associated with permeability orientations $\alpha_{i}$ relate to $\boldsymbol{\Phi}$ via diffeomorphisms $G_{i}$. This has the fundamental implication that all these maps exhibit (qualitatively) the same Lagrangian transport properties. (e.g. chaotic advection in one map implies this in all maps.) This property is of great practical relevance for the design of pumping schemes by admitting a tremendous reduction in effort for optimization of the reservoir throughflow; examination of subrange $0 \leq \alpha \leq \alpha_{\max }$, with $\alpha_{\max }=\Theta / j=2 \pi / N$ and integer $j$ according to Sect. 2.1, is for given pumping scheme and permeability ratio $\kappa$ namely sufficient.

Figure 13 demonstrates a typical relation between the Lagrangian dynamics for permeability orientations $\alpha_{i}$ by way of the previous pumping scheme $\left(\Theta, T_{\text {step }}\right)=(3 \pi / 4,0.2)$ and anisotropy $(\alpha, \kappa)=(0,5)$. Note that here an even $N$ (i.e. $N=8)$ implies $\alpha_{i+N / 2}=$ $\alpha_{i}+\pi=\alpha_{i}$ (due to the fact that invariance of the anisotropy to rotation by $\pi$ restricts the relevant range to $0 \leq \alpha \leq \pi)$ and thus $\boldsymbol{\Phi}^{[i+N / 2]}=\boldsymbol{\Phi}^{[i]}$. Hence, only $N / 2=4$ distinct cases occur. Moreover, a linear transformation between map $\boldsymbol{\Phi}$ and $\boldsymbol{\Phi}^{[7]}$ emerges from substitution of time-reversal symmetry (23), which is preserved for $\alpha=0$ (Sect. 5.3.2), in conjunction with its definition $S_{1}=S_{x} \boldsymbol{\Phi}_{1}$ into (31), yielding

$$
\boldsymbol{\Phi}^{[7]}=S_{3} \boldsymbol{\Phi}^{-1} S_{3}^{-1}, \quad S_{3}=R^{-1} S_{x},
$$

with $S_{3}$ a reflection about the $x$-axis and subsequent reorientation with angle $-\Theta$. This readily extends to other maps through relations $\boldsymbol{\Phi}^{[4]}=\boldsymbol{\Phi}$ and $\boldsymbol{\Phi}^{[3]}=\boldsymbol{\Phi}^{[7]}$.

Permeability orientations $\alpha=0$ and $\alpha=\pi / 2$ result in time-reversal symmetry $S_{1}$ according to (23) for $\boldsymbol{\Phi}$ (Sect. 5.3.2). This generalizes to maps $\boldsymbol{\Phi}^{[i]}$ for the associated $\alpha_{i}=i \Theta$ and $\alpha_{i}=\pi / 2+i \Theta$ upon substituting (31) in (23), yielding

$$
\boldsymbol{\Phi}^{[i]}=S_{[i]}\left(\boldsymbol{\Phi}^{[i]}\right)^{-1} S_{[i]}, \quad S_{[i]}=G_{i} S_{1} G_{i}^{-1},
$$

with $S_{[i]}=S_{[i]}^{-1}$. Thus alignment of any well pair with the permeability orientation leads to a time-reversal symmetry. Reconsider for illustration, e.g. pumping scheme $\left(\Theta, T_{\text {step }}\right)=$ $(3 \pi / 4,0.2)$ for permeability orientation $\alpha=\pi / 4$ shown in Fig. 10, which corresponds with 


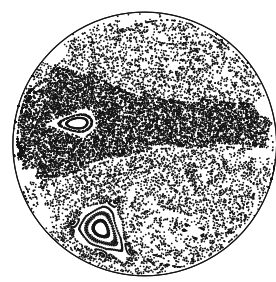

(a)

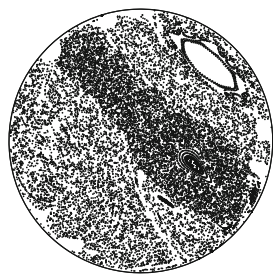

(b)

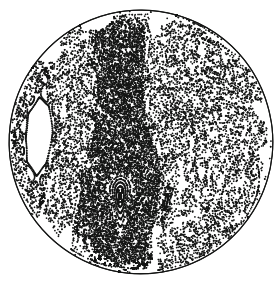

(c)

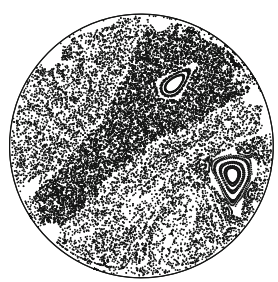

(d)

Fig. 13 Symmetry between permeability orientations according to (31) demonstrated for pumping scheme $\left(\Theta, T_{\text {step }}\right)=(3 \pi / 4,0.2)$ and $(\alpha, \kappa)=(0,5.0)$

$\boldsymbol{\Phi}^{[3]}=\boldsymbol{\Phi}^{[7]}$ in Fig. 13. Combining (23) and (32) readily yields time-reversal symmetry $S_{[3]}=S_{3} S_{1} S_{3}^{-1}$ for (33). The corresponding symmetry curve $S_{[3]} \mathcal{I}_{[3]}=\mathcal{I}_{[3]}$ can, consistent with reorientation (32), be shown to be given by $\mathcal{I}_{[3]}=S_{3} \mathcal{I}_{1}$, with $\mathcal{I}_{1}$ the symmetry curve of $S_{1}$ (Fig. 12).

The "dark" confinement regions within the chaotic sea in Fig. 13 for all maps $\boldsymbol{\Phi}^{[i]}$ concentrate around the corresponding symmetry curves $\mathcal{I}_{[i]}$ (not shown). This substantiates the earlier conjecture that this phenomenon intimately relates to time-reversal symmetries (33) and, inherently, to well alignment. Thus (temporary) alignment of the wells with the anisotropy (through a suitable well configuration and pumping scheme) in principle enables systematic confinement of production fluid for a pre-defined time window. An important practical application may exist in, e.g., heat extraction primarily from the confinement region so as to induce a global temperature gradient within the reservoir for the purpose of stimulating internal heat distribution. This may benefit overall heat recovery and performance.

\section{Conclusions}

The study investigates the impact of anisotropy on the distribution of production fluids in underground (geothermal) reservoirs by way of a 2D Darcy-type time-periodic flow in a circular reservoir driven by reoriented injector-producer wells. This study concentrates on fundamental properties of the Lagrangian transport in the reservoir (vs. well configurations and pumping schemes) with a particular focus on accomplishment of chaotic advection and, inherently, efficient fluid distribution. Such a scheme admits practical implementation by a static array of wells on the circumference of the reservoir that are pair-wise activated as described in Sect. 2.1.

Incompressibility implies a Hamiltonian structure for the Lagrangian equations of motion for any (an-)isotropic system. This has the fundamental consequence that chaotic advection always emanates from the breakdown of an essentially non-chaotic state (denoted "integrable state") that sets in for very high reorientation frequencies. Systems take this generic "route to chaos" upon lowering the reorientation frequency as illustrated in Sects. 4.1 and 5.1. Hence, pumping schemes with reorientation frequency below a certain upper bound is necessary (yet not sufficient) for global chaotic advection. This (in principle) enables systematic and efficient fluid distribution throughout the entire reservoir for optimal extraction of heat as well as other resources as oil, gas or (dissolved) minerals. Further practical applications of chaotic advection thus accomplished may be found in efficient subsurface distribution of injected substances as e.g. chemicals for in situ groundwater remediation and mining or $\mathrm{CO}_{2}$ for underground storage. 
However, subsurface transport other than chaotic advection may be equally useful for practical purposes. The formation of islands in the Poincaré sections for certain pumping schemes for instance offers a way to entrap and circulate (production) fluid in designated subregions of the reservoir. This may ,e.g., enable manipulation of internal heat distribution for the benefit of better thermal performance or greater sustainability of EGSs by creation of "heat sinks" through (temporary) entrapment and localized circulation of cold production fluid. Further applications of islands may exist in containment of contaminants within a subregion for its effective treatment during in situ groundwater remediation or creation of "reaction zones" for in situ mining. Islands can for such purposes be created in various shapes and arrangements by, e.g., operating an even number of wells at a high reorientation frequency (Sects. 4.2, 5.1). Moreover, given that coherent structures in Poincaré sections form instantaneously, changing the pumping scheme facilitates switching between different "transport modes".

Symmetries are key organizing mechanisms for the Lagrangian transport and thus play a vital role in the "route to chaos". Two kind of symmetries are relevant for the isotropic case: (i) symmetries between Poincaré sections of different sampling levels; (ii) symmetries within Poincaré sections of a given sampling level (Sect. 4.3). The former kind causes Poincaré sections associated with consecutive steps in the pumping scheme to relate via simple rotations. The latter kind causes coherent structures within Poincaré sections to be subject to multiple reflectional symmetries. Thus symmetries impose a high degree of ordering and coherence upon the Lagrangian dynamics.

Anisotropy typically breaks the above symmetries and thus brings about a fundamental reduction in ordering and coherence within/between Poincaré sections. This is consistent with the observation that anisotropy overall tends to diminish islands and promote chaos as demonstrated in Sect. 5.2. However, symmetries - and thus increased ordering/coherencenonetheless occur yet for non-generic cases. The simple rotational symmetry between Poincaré sections is, e.g., partially restored for pumping schemes employing an even number of injector-producer wells (Sect. 5.3.1). Moreover, one reflectional symmetry within Poincaré sections is preserved for well configurations aligned with the permeability orientation (Sect. 5.3.2). This symmetry appears crucial to the prolonged confinement of fluid to subregions of chaotic areas; this intriguing "order within chaos" namely is observed only for such well alignment. Further investigation to conclusively establish this connection is underway.

In anisotropic reservoirs, besides the pumping scheme itself, placement of the wells relative to the principal directions of anisotropy has great impact on the subsurface transport. Well configurations not aligned with the anisotropy breaks symmetries and thus promotes efficient fluid distribution by chaotic advection (Sect. 5.3). A configuration consisting of wells aligned with the anisotropy, on the other hand, promotes the entrapment and local circulation of production fluid by islands or confinement near symmetry axes (Sect. 5.3.2). Both chaotic advection and entrapment may have important practical applications such as those mentioned before. Hence, the arrangement of the (activated) wells in relation to the anisotropy is a further important design and control parameter for well configurations, pumping schemes and reservoir operation scenarios.

Anisotropy introduces a new kind of symmetry: symmetry between permeability orientations for a given well configuration. This property is key to the above symmetry associated with well alignment. Moreover, this symmetry is of great practical relevance for the design of pumping schemes by admitting a tremendous reduction in the parameter space that must be explored in optimization studies. An examination of subrange $0 \leq \alpha \leq \alpha_{\text {max }}$, with $\alpha_{\max }=2 \pi / N$ as opposed to $\alpha_{\max }=2 \pi$, is for given pumping scheme and permeability 
ratio $\kappa$ namely sufficient as chaotic advection in maps for permeability direction $\alpha$ implies chaotic advection for the direction $(\alpha+i \Theta)$ with integer $i$ as per Sect. 5.3.3, since all these maps exhibit (qualitatively) the same Lagrangian transport properties.

Open Access This article is distributed under the terms of the Creative Commons Attribution 4.0 International License (http://creativecommons.org/licenses/by/4.0/), which permits unrestricted use, distribution, and reproduction in any medium, provided you give appropriate credit to the original author(s) and the source, provide a link to the Creative Commons license, and indicate if changes were made.

\section{Appendix: Integrable State}

The integrable state of system (14) can be shown to correspond with limit $T_{\text {step }}=0^{+}$. Express to this end stroboscopic map (12) as the flow by a steady velocity field $\bar{v}$ following

$$
\boldsymbol{x}_{1}=\boldsymbol{\Phi}\left(\boldsymbol{x}_{0}\right)=\boldsymbol{x}_{0}+T \overline{\boldsymbol{v}}\left(\boldsymbol{x}_{0}\right), \quad \overline{\boldsymbol{v}}\left(\boldsymbol{x}_{0}\right)=\frac{1}{T} \int_{0}^{\mathrm{T}} \boldsymbol{v}\left[\boldsymbol{\Phi}_{t}\left(\boldsymbol{x}_{0}\right), t\right] \mathrm{d} t,
$$

with $\overline{\boldsymbol{v}}\left(\boldsymbol{x}_{0}\right)$ the period-wise Lagrangian average of the velocity field along trajectories $\boldsymbol{x}(t)=$ $\boldsymbol{\Phi}_{t}\left(\boldsymbol{x}_{0}\right)$. This Lagrangian average, through composite form (8), admits expression in terms of the step-wise flows $\boldsymbol{v}_{k}$ and base flow $\boldsymbol{v}_{1}$ via

$$
\overline{\boldsymbol{v}}\left(\boldsymbol{x}_{0}\right)=\frac{1}{T} \sum_{k=1}^{N} \int_{0}^{T_{\text {step }}} \boldsymbol{v}_{k}\left[\boldsymbol{\Phi}_{t}\left(\tilde{\boldsymbol{x}}_{k}\right)\right] \mathrm{d} t=\frac{1}{T} \sum_{k=1}^{N} \int_{0}^{T_{\text {step }}} \boldsymbol{v}_{1}\left[R^{1-k} \boldsymbol{\Phi}_{t}\left(\tilde{\boldsymbol{x}}_{k}\right)\right] \mathrm{d} t,
$$

with $\tilde{\boldsymbol{x}}_{k}$ the step-wise initial tracer position [i.e. $\tilde{\boldsymbol{x}}_{1}=\boldsymbol{x}_{0}$ and $\tilde{\boldsymbol{x}}_{k+1}=\tilde{\boldsymbol{x}}_{k}+\varepsilon \boldsymbol{v}_{k}\left(\widetilde{\boldsymbol{x}}_{k}\right)$ ]. Important in the present context is that the Lagrangian-averaged field $\bar{v}$, though generically non-solenoidal (i.e. $\nabla \cdot \overline{\boldsymbol{v}} \neq 0$ ), tends towards a solenoidal field for diminishing $T_{\text {step }}$. This follows from exploiting the fact that map $\boldsymbol{\Phi}$ is volume-preserving, implying

$$
\left|\frac{\partial \boldsymbol{\Phi}}{\partial \boldsymbol{x}_{0}}\right|=1=\left|\boldsymbol{I}+T \frac{\partial \overline{\boldsymbol{v}}}{\partial \boldsymbol{x}_{0}}\right|=1+T \nabla_{0} \cdot \overline{\boldsymbol{v}}+T^{2}\left|\frac{\partial \overline{\boldsymbol{v}}}{\partial \boldsymbol{x}_{0}}\right|,
$$

where $\nabla_{0}=\partial / \partial \boldsymbol{x}_{0}$, and thus yielding

$$
\nabla_{0} \cdot \overline{\boldsymbol{v}}=-T\left|\frac{\partial \overline{\boldsymbol{v}}}{\partial \boldsymbol{x}_{0}}\right|=-N T_{\text {step }}\left|\frac{\partial \overline{\boldsymbol{v}}}{\partial \boldsymbol{x}_{0}}\right| \sim \mathcal{O}\left(T_{\text {step }}\right),
$$

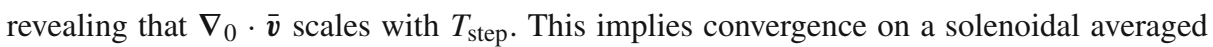
velocity — and thus a Hamiltonian structure—for diminishing $T_{\text {step }}$, i.e.

$$
\lim _{T_{\text {step }} \rightarrow \varepsilon} \nabla_{0} \cdot \overline{\boldsymbol{v}}=\varepsilon \Rightarrow \lim _{T_{\text {step }} \rightarrow \varepsilon} \overline{\boldsymbol{v}}=\left(\frac{\partial \bar{H}}{\partial y},-\frac{\partial \bar{H}}{\partial x}\right)+\mathcal{O}(\varepsilon),
$$

with $\varepsilon$ very close to (yet essentially different from) zero.

Hamiltonian $\bar{H}$ emanates from the Hamiltonians $H_{k}$ associated with the step-wise flows $\boldsymbol{v}_{k}$ as follows. Average velocity (35) simplifies in the limit case to

$$
\begin{aligned}
\lim _{T_{\text {step }} \rightarrow \varepsilon} \overline{\boldsymbol{v}}\left(\boldsymbol{x}_{0}\right) & =\lim _{T_{\text {step }} \rightarrow \varepsilon} \frac{1}{N T_{\text {step }}} \sum_{k=1}^{N} \int_{0}^{T_{\text {step }}} \boldsymbol{v}_{k}\left[\boldsymbol{\Phi}_{t}\left(\tilde{\boldsymbol{x}}_{k}\right)\right] \mathrm{d} t \\
& =\frac{1}{N} \sum_{k=1}^{N} \boldsymbol{v}_{k}\left(\widetilde{\boldsymbol{x}}_{k}\right)=\frac{1}{N} \sum_{k=1}^{N} \boldsymbol{v}_{1}\left(R^{1-k} \widetilde{\boldsymbol{x}}_{k}\right),
\end{aligned}
$$


due to $\lim _{T_{\text {step }} \rightarrow \varepsilon} \int_{0}^{T_{\text {step }}} f(t) \mathrm{d} t=f(0) T_{\text {step. }}$. This readily yields

$$
\bar{H}\left(\boldsymbol{x}_{0}\right)=\int\left[-\bar{v}_{y} \mathrm{~d} x+\bar{v}_{x} \mathrm{~d} y\right]=\frac{1}{N} \sum_{k=1}^{N} H_{k}\left(\tilde{\boldsymbol{x}}_{k}\right)=\frac{1}{N} \sum_{k=1}^{N} H_{1}\left[R^{1-k} \tilde{\boldsymbol{x}}_{k}\right],
$$

as corresponding Hamiltonian.

Important in the above is that the Lagrangian average for essentially nonzero $\varepsilon=0^{+}$ constitutes the integrable state and not the strict limit $\varepsilon=0$. The latter causes the Lagrangianaveraged velocity to coincide with the Eulerian average

$$
\overline{\boldsymbol{v}}_{E}\left(\boldsymbol{x}_{0}\right)=\frac{1}{N} \sum_{k=1}^{N} \boldsymbol{v}_{k}\left(\boldsymbol{x}_{0}\right)=\frac{1}{N} \sum_{k=1}^{N} \boldsymbol{v}_{1}\left(R^{1-k} \boldsymbol{x}_{0}\right),
$$

which closely agrees with the Lagrangian counterpart for odd $N$ yet vanishes identically for even $N$ on account of mutual cancellation of source-sink pairs. Hence, $\varepsilon=0$ strictly is a singular limit. For odd $N$ this is inconsequential in that the Eulerian average is non-trivial and thus closely approximates the true limit case $\varepsilon=0^{+}$(explaining validity of this ansatz in Metcalfe et al. 2010; Speetjens et al. 2006). However, for even $N$ the Eulerian average is trivial and fails to capture the integrable state.

\section{References}

Aref, H.: Stirring by chaotic advection. J. Fluid Mech. 143, 1-21 (1984)

Aref, H.: The development of chaotic advection. Phys. Fluids 14(4), 1315-1325 (2002)

Bolster, D., Valdés-Parada, F.J., LeBorgne, T., Dentz, M., Carrera, J.: Mixing in confined stratified aquifers. J. Contam. Hydrol. 120, 198-212 (2011)

Brown, D.W., Duchane, D.V., Heiken, G., Hriscu, V.T.: Mining the Earth's Heat: Hot Dry Rock Geothermal Energy. Springer Science \& Business Media, Berlin (2012)

Chen, D., Wyborn, D.: Habanero field tests in the Cooper Basin, Australia: a proof-of-concept for EGS. Geotherm. Res. Counc. Trans. 33(1), 159-164 (2009)

DiPippo, R.: Geothermal Power Plants. Butterworth-Heinemann, Oxford (2008)

Fang, Y., Elsworth, D., Cladouhos, T.T.: Estimating in-situ permeability of stimulated EGS reservoirs using MEQ moment magnitude: an analysis of Newberry MEQ data. In: Proceedings, 40th Workshop on Geothermal Reservoir Engineering, Stanford University (2015)

Gérard, A., Genter, A., Kohl, T., Lutz, P., Rose, P., Rummel, F.: The deep EGS (enhanced geothermal system) project at Soultz-sous-Forêts (Alsace, France). Geothermics 35(5), 473-483 (2006)

Grant, M.A.: Geothermal Reservoir Engineering. Academic Press, San Diego (2011)

Grant, M.A., Garg, S.K.: Recovery factor for EGS. In: Proceedings, 37th Workshop on Geothermal Reservoir Engineering, Stanford University, pp. 738-740 (2012)

Hidalgo, J.J., Carrera, J., Dentz, M.: Steady state heat transport in 3D heterogeneous porous media. Adv. Water Resour. 32(8), 1206-1212 (2009)

Horne, R.N.: Effects of Water Injection into Fractured Geothermal Reservoirs: A Summary of Experience Worldwide. Technical report, Stanford Geothermal Program (1982)

Hurter, S., Schellschmidt, R.: Atlas of geothermal resources in Europe. Geothermics 32(4), 779-787 (2003)

Kosack, C., Vogt, C., Marquart, G., Clauser, C., Rath, V.: Stochastic permeability estimation for the Soultz-sousforêts EGS reservoir. In: Proceedings, 36th Workshop on Geothermal Reservoir Engineering, Stanford University (2011)

Kreyszig, E.: Advanced Engineering Mathematics. Wiley, Chichester (1999)

Lester, D., Metcalfe, G., Trefry, M., Ord, A., Hobbs, B., Rudman, M.: Lagrangian topology of a periodically reoriented potential flow: symmetry, optimization, and mixing. Phys. Rev. E 80(3), 036208 (2009)

Li, M., Lior, N.: Analysis of hydraulic fracturing and reservoir performance in enhanced geothermal systems. J. Energy Resour. Technol. 137(4), 041203 (2015)

Massiot, C., McNamara, D.D., Nicol, A., Townend, J.: Fracture width and spacing distributions from borehole televiewer logs and cores in the Rotokawa geothermal field, New Zealand. In: Proceedings World Geothermal Congress (2015) 
Metcalfe, G., Lester, D., Ord, A., Kulkarni, P., Rudman, M., Trefry, M., Hobbs, B., Regenaur-Lieb, K., Morris, J.: An experimental and theoretical study of the mixing characteristics of a periodically reoriented irrotational flow. Philos. Trans. R. Soc. A 368(1918), 2147-2162 (2010)

Metcalfe, G., Speetjens, M., Lester, D., Clercx, H., et al.: Beyond passive: chaotic transport in stirred fluids. Adv. Appl. Mech. 45(109), 2012 (2012)

Nield, D.A., Bejan, A.: Convection in Porous Media. Springer Science \& Business Media, Berlin (2006)

Ott, E.: Chaos in Dynamical Systems. Cambridge University Press, Cambridge (2002)

Ottino, J., Jana, S., Chakravarthy, V.: From Reynolds's stretching and folding to mixing studies using horseshoe maps. Phys. Fluids 6(2), 685-699 (1994)

Ottino, J.M.: The Kinematics of Mixing: Stretching, Chaos, and Transport. Cambridge University Press, Cambridge (1989)

Piscopo, A.N., Neupauer, R.M., Mays, D.C.: Engineered injection and extraction to enhance reaction for improved in situ remediation. Water Resour. Res. 49(6), 3618-3625 (2013)

Pruess, K.: Enhanced geothermal systems (EGS) using $\mathrm{CO}_{2}$ as working fluid-A novel approach for generating renewable energy with simultaneous sequestration of carbon. Geothermics 35(4), 351-367 (2006)

Randolph, J.B., Saar, M.O.: Impact of reservoir permeability on the choice of subsurface geothermal heat exchange fluid: $\mathrm{CO}_{2}$ versus water and native brine. In: Proceedings for the Geothermal Resources Council 35th Annual Meeting, pp. 23-26 (2011)

Speetjens, M., Clercx, H., Van Heijst, G.: A numerical and experimental study on advection in threedimensional Stokes flows. J. Fluid Mech. 514, 77-105 (2004)

Speetjens, M., Metcalfe, G., Rudman, M.: Topological mixing study of non-Newtonian duct flows. Phys. Fluids 18(10), 103103 (2006)

Trefry, M.G., Lester, D.R., Metcalfe, G., Ord, A., Regenauer-Lieb, K.: Toward enhanced subsurface intervention methods using chaotic advection. J. Contam. Hydrol. 127(1), 15-29 (2012)

van Essen, G., Zandvliet, M., Van den Hof, P., Bosgra, O., Jansen, J.D.: Robust water flooding optimization of multiple geological scenarios. SPE J. 14(01), 202-210 (2009)

Wang, X., Wang, J., Wang, C., Zeng, L., Liu, X.: Quantitative description of characteristics of high-capacity channels in unconsolidated sandstone reservoirs using in situ production data. Pet. Sci. 7(1), 106-111 (2010)

Xing, H., Zhang, J., Liu, Y., Mulhaus, H.: Enhanced geothermal reservoir simulation. In: Proceedings of the Australian Geothermal Energy Conference, pp. 187-190 (2009)

Zimmermann, G., Reinicke, A.: Hydraulic stimulation of a deep sandstone reservoir to develop an enhanced geothermal system: laboratory and field experiments. Geothermics 39(1), 70-77 (2010) 